\title{
A enfermagem na prevenção e cuidados relacionados à pneumonia associada à ventilação mecânica: Uma revisão integrativa
}

\author{
Nursing in the prevention and care of ventilator-associated pneumonia: An integrative review \\ Enfermería en la prevención y atención de la neumonía asociada al ventilador: Una revisión
}

integradora

Recebido: 08/06/2021 | Revisado: 15/06/2021 | Aceito: 17/06/2021 | Publicado: 24/06/2021

Lidiane do Socorro Carvalho dos Santos

ORCID: https://orcid.org/0000-0002-6549-5986

Centro Universitário Metropolitano da Amazônia, Brasil

E-mail: lidianecarvalho90@yahoo.com.br

Silvani Damasceno de Barros

ORCIID: https://orcid.org/0000-0002-4752-6312

Centro Universitário Metropolitano da Amazônia, Brasil

E-mail: silvanedamasceno123@gmail.com

Milena Farah Damous Castanho Ferreira

ORCID: https://orcid.org/0000-0002-0645-2046

Centro Universitário Metropolitano da Amazônia, Brasil

Email: milenafcastanho@hotmail.com

Brenda Tanielle Dutra Barros

ORCID: https://orcid.org/0000-0002-3184-050X

Centro Universitário Metropolitano da Amazônia, Brasil

Email: brendatanielle.enf@gmail.com

Rômulo Leno Miranda Barros

ORCID: https://orcid.org/0000-0003-1802-4521

Universidade da Amazônia, Brasil

E-mail: romuloleno@hotmail.com

Beatriz Rocha Barata de Souza

ORCID: https://orcid.org/0000-0002-3796-4626

Centro Universitário Metropolitano da Amazônia, Brasil

E-mail: bdbeatriz1@ hotmail.com

José Eduardo Resende Campos

ORCID: https://orcid.org/0000-0002-4070-8543

Centro Universitário Metropolitano da Amazônia, Brasil

E-mail: educamposjrc@gmail.com

Maria Barbara Freire Lameira

ORCID: https://orcid.org/0000-0002-5386-7308

Centro Universitário Metropolitano da Amazônia, Brasil

E-mail: barbarafreirelameira@gmail.com

Marildete da Conceição Paula

ORCID: https://orcid.org/0000-0001-9092-4693

Centro Universitário Metropolitano da Amazônia, Brasil

E-mail: marypaula72@ hotmail.com

Maria de Nazare Gonçalves Pereira Ferreira

ORCID: https://orcid.org/0000-0003-0784-4866

Centro Universitário Metropolitano da Amazônia, Brasil

E-mail: nazarepereira480@yahoo.com.br

Letícia Erica Neves dos Prazeres

ORCID: https://orcid.org/0000-0002-8155-2960

Centro Universitário Metropolitano da Amazônia, Brasil

E-mail: letneves@ outlook.com

Fábio Ramon de Oliveira Lobato

ORCID: https://orcid.org/0000-0002-7078-6817

Universidade do Estado do Pará, Brasil

E-mail: fabioolibato@gmail.com

Raimunda Nonata Silva de Almeida

ORCID: https://orcid.org/0000-0003-2453-902X

Faculdade Paraense de Ensino, Brasil

E-mail: nonataalmeida7@gmail.com

Ana Carolina Cunha Gama

ORCID: https://orcid.org/0000-0003-4728-1156

Centro Universitário Metropolitano da Amazônia, Brasil

E-mail: carol.amsp@hotmail.com 
Tatiana Fabíola da Silva Lima

ORCID: https://orcid.org/0000-0001-5966-9727

Universidade do Estado do Pará, Brasil

E-mail: tatiana.fs.lima2@gmail.com

\begin{abstract}
Resumo
A Ventilação Mecânica Invasiva (VMI) é um tratamento ventilatório artificial que é utilizado nas Unidades de Terapia Intensiva (UTI) em casos graves de insuficiência respiratória aguda (IRA) ou quando o paciente não consegue realizar as trocas gasosas de forma adequada, mesmo havendo a suplementação de oxigênio. O objetivo desta revisão foi descrever, a partir das evidências científicas encontradas na literatura, sobre cuidados de enfermagem ao paciente crítico, na prevenção e tratamento relacionado à pneumonia associada à ventilação mecânica (PAV). Trata-se de uma Revisão Integrativa, elaborada através das buscas realizadas nas bases científicas: Banco de Dados em Enfermagem (BDENF), Literatura Latino-Americana e do Caribe em Ciências da Saúde (LILACS) e Scientific Electronic Library Online (SCIELO), utilizando as palavras-chave: "Pneumonia", "Enfermagem" e "Unidade de Terapia Intensiva", sendo incluídos os estudos publicados no período de 2011 à 2020; foram agrupados (52) artigos em categorias temáticas mediante as diferentes abordagens dos estudos quanto ao processo de pneumonia associada à ventilação mecânica, totalizando amostra final composta por (12) artigos através do modelo descritivo com abordagem qualitativa. Evidenciou-se que a atuação do enfermeiro no cuidado prestado à PAV é de extrema importância uma vez que a assistência qualificada, um bom relacionamento multiprofissional e a implementação de pacotes de cuidados são as medidas mais recomendadas na literatura, pois demonstra uma melhor eficiência na assistência, favorecendo então uma diminuição nas taxas de incidência desse acometimento.
\end{abstract}

Palavras-chave: Pneumonia; Enfermagem; Unidade de terapia intensiva.

\begin{abstract}
Invasive Mechanical Ventilation (IMV) is an artificial ventilatory treatment that is used in the Intensive Care Units (ICU) in severe cases of acute respiratory failure (ARI) or when the patient is unable to perform gas exchange properly, even with the oxygen supplementation. The aim of this review was to describe, based on the scientific evidence found in the literature, about nursing care for critically ill patients, in the prevention and treatment of ventilator-associated pneumonia (VAP). This is an integrative review, elaborated through searches carried out in the scientific databases: Database in Nursing (BDENF), Latin American and Caribbean Literature in Health Sciences (LILACS) and Scientific Electronic Library Online (SCIELO), using the keywords: "Pneumonia", "Nursing" and "Intensive Care Unit", including studies published from 2011 to 2020; (52) articles were grouped into thematic categories according to the different approaches of the studies regarding the process of pneumonia associated with mechanical ventilation, totaling a final sample consisting of (12) articles through the descriptive model with a qualitative approach. It was evident that the role of nurses in the care provided to VAP is extremely important, since qualified care, a good multidisciplinary relationship and the implementation of care packages are the most recommended measures in the literature, as it demonstrates better efficiency in care, thus favoring a decrease in the incidence rates of this involvement.
\end{abstract}

Keywords: Pneumonia; Nursing; Intensive care unit.

\title{
Resumen
}

La Ventilación Mecánica Invasiva (VMI) es un tratamiento ventilatorio artificial que se utiliza en las Unidades de Cuidados Intensivos (UCI) en casos severos de insuficiencia respiratoria aguda (IRA) o cuando el paciente no puede realizar el intercambio gaseoso correctamente, incluso con la suplementación de oxígeno. El objetivo de esta revisión fue describir, con base en la evidencia científica encontrada en la literatura, sobre la atención de enfermería al paciente crítico, en la prevención y tratamiento de la neumonía asociada al ventilador (NAV). Se trata de una revisión integradora, desarrollada a través de búsquedas realizadas en las bases de datos científicas: Base de Datos en Enfermería (BDENF), Literatura Latinoamericana y del Caribe en Ciencias de la Salud (LILACS) y Biblioteca Electrónica Científica en Línea (SCIELO), utilizando las palabras clave: "Neumonía". , "Enfermería" y "Unidad de Cuidados Intensivos", incluidos los estudios publicados de 2011 a 2020; (52) artículos se agruparon en categorías temáticas de acuerdo a los diferentes abordajes de los estudios sobre el proceso de neumonía asociada a ventilación mecánica, totalizando una muestra final conformada por (12) artículos a través del modelo descriptivo con abordaje cualitativo. Se evidenció que el rol del enfermero en la atención brindada a la NAV es sumamente importante, ya que la atención calificada, una buena relación multidisciplinar y la implementación de paquetes de atención son las medidas más recomendadas en la literatura, ya que demuestra mayor eficiencia en la atención, por lo que favoreciendo una disminución en las tasas de incidencia de esta afectación.

Palabras clave: Neumonía; Enfermería; Unidad de terapia intensiva. 


\section{Introdução}

A Ventilação Mecânica Invasiva (VMI) é um tratamento ventilatório artificial que é utilizado nas Unidades de Terapia Intensiva (UTI) em casos graves de insuficiência respiratória aguda (IRA) e quando ocorre a necessidade em que o paciente não consegue realizar as trocas gasosas de forma adequada mesmo havendo a suplementação de oxigênio (TubertBrohman et al., 2013). A Pneumonia Associada à Ventilação Mecânica (PAVM) é a principal e a mais importante infecção que afeta pacientes críticos na Unidade de Terapia Intensiva (UTI), devido a sua vulnerabilidade, é geralmente de origem aspirativa, seguida de refluxo gastrointestinal (Rodrigues et al., 2016).

Segundo a ANVISA alguns dos fatores de risco associados a PAVM são: a idade avançada, a intubação e reintubação, tempo prolongado de ventilação mecânica, dentre outros. Até 33\% desses pacientes vão a óbito em decorrência direta desses fatores (Rodrigues et al. 2016).

É necessária uma equipe multi e interdisdisciplinar, com recursos humanos suficientes para suprir a demanda do local com qualidade, ou seja, toda uma gama de pessoas cuidando do espaço físico, com o objetivo ofertar uma assistência qualificada ao usuário (Prazeres et al., 2021; Neto et al., 2016). Mediante a essas preocupações, para prevenir a ocorrência da PAV em UTI's foi criado pelo Institute for Health Care Impravement (IHI) o bundle de ventilação ou pacotes de cuidados, onde foram estabelecidas medidas para a prevenção da PAV baseados em evidências científicas (Tubert-Brohman et al., 2013).

Alguns desses cuidados para o controle de infecção são: educação dos profissionais de saúde, lavagem das mãos e uso das luvas quando necessário, evitar acúmulo de secreções, evitar uso de equipamentos entre pacientes, proceder a desinfecção adequada do equipamento respiratório, umidificar o ventilador com água estéril, assim como fazer a manutenção da cabeceira da cama do paciente entre 30 e 45 graus para reduzir o risco de aspiração, dessa forma contribui para a redução do índice de pneumonia associada à ventilação mecânica (Farias et al., 2009).

Este estudo torna-se relevante, pois, entre os profissionais da saúde mais diretamente envolvidos na assistência, o enfermeiro ocupa um espaço estratégico e de referência na equipe, uma vez que, também é função do enfermeiro barrar a cadeia de transmissão de microrganismos, diminuindo o risco de infecções relacionadas a assistência à saúde (IRAS) e melhorando a qualidade da assistência e a segurança do paciente e do profissional (Silva et al., 2017).

Sistematizar tais publicações científicas permitirá a identificação dos estudos realizados e debatidos sobre a prevenção e assistência de enfermagem a pacientes em ventilação mecânica que tem o risco ou desenvolveram a PAV, com isso identificar como os enfermeiros têm realizado suas condutas preventivas e terapêuticas quanto a esse importante problema no contexto das Unidades de Terapias Intensivas.

Para tanto, os autores traçam a seguinte questão de pesquisa: Quais os principais achados, descritos nas evidencias científicas na literatura sobre os cuidados de enfermagem ao paciente crítico, na prevenção e tratamento relacionado à pneumonia associada à ventilação mecânica no período de 2011 à 2020 ?

A pesquisa tem como objetivo descrever os principais achados nas evidências científicas, sobre os cuidados de enfermagem ao paciente crítico, na prevenção e tratamento relacionado à pneumonia associada à ventilação mecânica no período de 2011 à 2020.

\section{Metodologia}

Trata-se de um estudo bibliográfico, do tipo Revisão Integrativa (RI), através do modelo descritivo, com abordagem qualitativa.

A RI é um método que permite síntese de conhecimento por meio de processo sistemático e rigoroso (Mendes, Silveira \& Galvão, 2019). A abordagem qualitativa responde a questões muito particulares. Com preocupações nas ciências sociais, com o nível de realidade que não pode ser quantificado (Minayo, 2001). 
Para a construção da pesquisa utilizou-se das seis fases da RI, sendo elas: Definição de qual será Pergunta da Revisão; Busca e escolha dos dados que serão utilizados; extrair a demanda de dados dos estudos e categorização; Análise rígida dos estudos incluídos; Síntese do produto obtido da Revisão; Demonstração da revisão integrativa, por meio da síntese do conhecimento (Souza, Silva \& Carvalho, 2010).

Definiu-se como questão norteadora: Quais os principais achados nas evidências científicas na literatura sobre os cuidados de enfermagem ao paciente crítico, na prevenção e tratamento relacionado à pneumonia associada à ventilação mecânica no período de 2011 à 2020, período que possui grande produção científica sobre a temática e que se faz atual.

As buscas de evidências foram realizadas nas bases Banco de Dados em Enfermagem (BDENF), Literatura LatinoAmericana e do Caribe em Ciências da Saúde (LILACS) e Scientific Electronic Library Online (SCIELO). Foi utilizado as seguintes palavras-chave: "Pneumonia", "Enfermagem" e "Unidade de Terapia Intensiva". Como critério de inclusão foram selecionados: artigos completos, teses, monografias e dissertações publicadas em fontes online referentes ao período de 2011 à 2020, em língua portuguesa com abordagem ao tema de estudo. Foram excluídos: textos incompletos, publicação em língua estrangeiras, fora do período, sem afinidade com o tema.

Para visualização, sistematização e análise da pesquisa, foi utilizado o fluxograma PRISMA (Principais Itens para Relatar Revisões sistemáticas e Meta-análises). Tal fluxograma (figura 1) permite visualizar a utilização de filtros de seleção da pesquisa e com isso auxilia na sistematização da análise dos artigos, além disso, possibilita uma análise criteriosa dos relatos pesquisados nas bases de dados (Galvão, Pansani \& Harrad, 2015).

A coleta de dados foi realizada utilizando o formulário adaptado de Ursi \& Galvão (2006) que possibilita extrair informações dos artigos permitindo analisar individualmente cada artigo metodológico. No (quadro 1) está contido a os dados acadêmicos dos estudos e no (quadro 2) a avaliação crítica dos estudos incluídos, além de estarem classificados por meio de uma hierarquia das evidências segundo o delineamento da pesquisa: Nível 1 Evidências resultantes da meta-análise; Nível 2 Evidências com delineamento experimental; Nível 3 Evidências de estudo quase-experimental; Nível 4 Evidências de estudo não-experimental ou com abordagem qualitativa; Nível 5 Evidências de relatos de casos ou de experiência; Nível 6 Evidências com opiniões de especialistas (Soares et al., 2014).

Os dados foram analisados de acordo com a análise de conteúdo de Bardin, a qual se divide em três etapas: préanálise, exploração do material e resultados obtidos e interpretação dos dados. A pré-análise que consiste na fase de organização, leitura flutuante, exploração do material ou codificação e tratamento dos resultados. A exploração do material tem o objetivo de analisar e busca de organização da ideia central. Na terceira fase é estabelecido as interpretações conforme o objetivo do trabalho (Teixeira et al., 2013).

\section{Resultados}

A coleta de dados foi realizada entre agosto à novembro de 2020. A princípio foram identificadas 52 evidências científicas nas bases de dados BDENF, LILACS e SciELO, porém, após a identificação dos artigos duplicados restaram 39. Foi aplicado filtros com bases no critério de exclusão como: textos incompletos, resumos, em língua estrangeira, publicações fora do período definido, sem afinidade com o tema que foi reduzindo o número de artigos selecionados. Por último foi realizada a leitura detalhada dos artigos, identificado 12 artigos que possuem direta relação com o tema e com a questão de pesquisa, sendo encontrados 5 na BDENF, 5 na LILACS e 2 na SCIELO.

Para demonstrar este processo, foi utilizado o Fluxograma PRISMA a seguir: 
Research, Society and Development, v. 10, n. 7, e58210716935, 2021

(CC BY 4.0) | ISSN 2525-3409 | DOI: http://dx.doi.org/10.33448/rsd-v10i7.16935

Fluxograma 1 - Processo de eleição seguindo o método PRISMA.
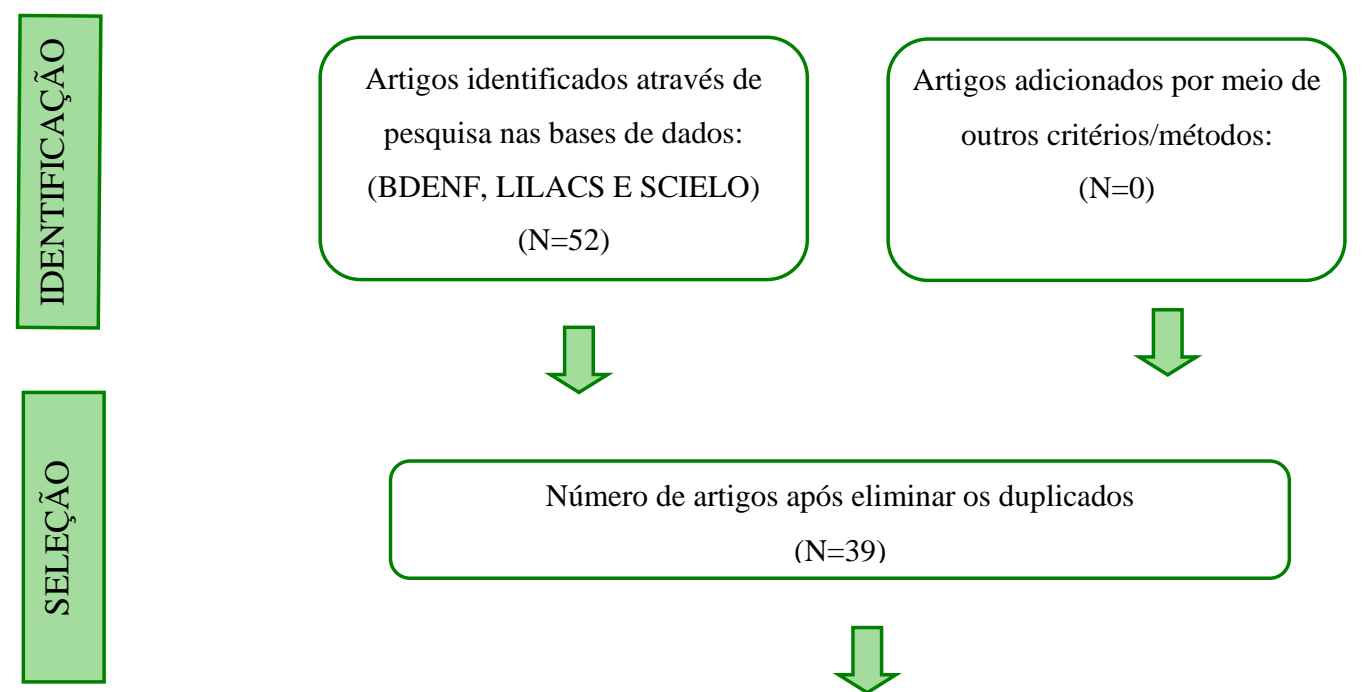

Número de artigos após eliminar os duplicados

$(\mathrm{N}=39)$
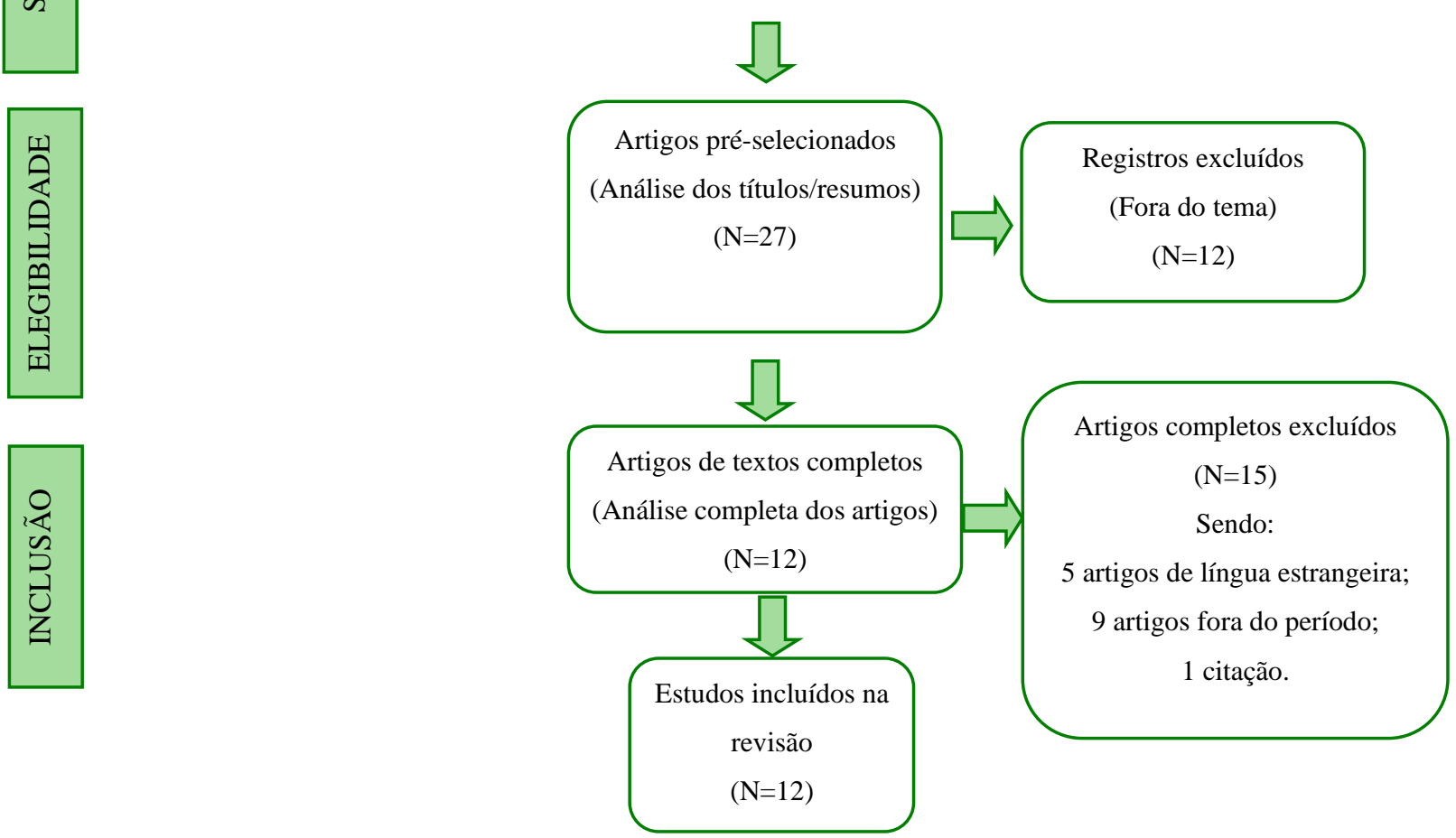

Fonte: Autores.

A distribuição dos artigos foi enquadrada quanto as variáveis: bases de dados, título do artigo, título do periódico, autores e ano de publicação estão representadas no Quadro 1. 
Quadro 1 - SÍNTESE 1. Caracterização dos estudos.

\begin{tabular}{|c|c|c|c|c|}
\hline $\begin{array}{l}\text { BASE DE } \\
\text { DADOS }\end{array}$ & TÍTULO DO ARTIGO & $\begin{array}{l}\text { TÍTULO DO } \\
\text { PERIÓDICO }\end{array}$ & AUTORES & $\begin{array}{l}\text { ANO DE } \\
\text { PUBLICAÇÃO }\end{array}$ \\
\hline SCIELO & $\begin{array}{l}\text { Eficácia de estratégias educativas para ações } \\
\text { preventivas da pneumonia associada à ventilação } \\
\text { mecânica. }\end{array}$ & $\begin{array}{l}\text { Rev Enferm Anna } \\
\text { Nery }\end{array}$ & $\begin{array}{l}\text { Gonçalves, F A. F. et } \\
\text { al. }\end{array}$ & 2012 \\
\hline SCIELO & $\begin{array}{l}\text { Boas práticas na prevenção de pneumonia associada à } \\
\text { ventilação mecânica }\end{array}$ & $\begin{array}{l}\text { Acta Paulista de } \\
\text { Enfermagem }\end{array}$ & $\begin{array}{l}\text { Alecrim, R. X. } \\
\text { et al. }\end{array}$ & 2019 \\
\hline LILACS & $\begin{array}{l}\text { Adesão às medidas preventivas versus incidência de } \\
\text { pneumonia associada à ventilação mecânica }\end{array}$ & $\begin{array}{c}\text { Revista de } \\
\text { Epidemiologia e } \\
\text { Controle de Infecção }\end{array}$ & $\begin{array}{l}\text { Lourençone, E. M. S. } \\
\text { et al. }\end{array}$ & 2019 \\
\hline LILACS & $\begin{array}{l}\text { Caracterização clínica dos pacientes sob ventilação } \\
\text { mecânica internados em unidade de terapia intensiva }\end{array}$ & Rev Cuid. & Barros, F. R. B. & 2019 \\
\hline LILACS & $\begin{array}{l}\text { Pneumonia Associada à Ventilação Mecânica: } \\
\text { Conhecimento dos Profissionais de Saúde Acerca da } \\
\text { Prevenção e Medidas Educativas }\end{array}$ & $\begin{array}{l}\text { Rev Fund Care } \\
\text { Online. }\end{array}$ & Melo, M. M. et al. & 2019 \\
\hline LILACS & $\begin{array}{l}\text { Caracterização clínica dos pacientes sob ventilação } \\
\text { mecânica internados em unidade de terapia intensiva }\end{array}$ & Rev Fund Care Online & Ribeiro, C. L. et al. & 2018 \\
\hline LILACS & $\begin{array}{l}\text { Prevalência de pneumonia associada à ventilação } \\
\text { mecânica por meio de análise das secreções } \\
\text { traqueobrônquicas }\end{array}$ & Rev Rene & Ferreira, E. G. et al. & 2017 \\
\hline BDENF & $\begin{array}{l}\text { Adesão ao protocolo de pneumonia associado } \\
\text { àventilação mecânica }\end{array}$ & $\begin{array}{l}\text { Rev enferm UFPE } \\
\text { online }\end{array}$ & Zigart, J. A. A. et al. & 2019 \\
\hline BDENF & $\begin{array}{l}\text { Fatores de risco que favorecem a pneumonia associada } \\
\text { à ventilação mecânica }\end{array}$ & $\begin{array}{l}\text { Rev enferm UFPE } \\
\text { online }\end{array}$ & Santos, C. R. et al & 2018 \\
\hline BDENF & $\begin{array}{l}\text { Pneumonia associada à ventilação mecânica: } \\
\text { Medidas preventivas conhecidas pelo enfermeiro }\end{array}$ & $\begin{array}{l}\text { Revista Baiana de } \\
\text { Enfermagem }\end{array}$ & $\begin{array}{l}\text { Moreira, B. S. G. et } \\
\text { al. }\end{array}$ & 2011 \\
\hline BDENF & $\begin{array}{l}\text { Pneumonia associada à ventilação mecânica: } \\
\text { percepção dos profissionais de enfermagem }\end{array}$ & $\begin{array}{l}\text { Rev enferm UFPE } \\
\text { online }\end{array}$ & Dutra, L. A. et al. & 2019 \\
\hline BDENF & $\begin{array}{l}\text { Valoração e registros sobre higiene oral de pacientes } \\
\text { intubados nas } \\
\text { Unidades de Terapia Intensiva }\end{array}$ & $\begin{array}{l}\text { REME }- \text { Rev Min } \\
\text { Enferm }\end{array}$ & Zanei, S. S. V. & 2016 \\
\hline
\end{tabular}

Fonte: Autores.

A metodologia, os objetivos dos estudos e seus resultados e nível de evidência, foram analisados e explorados para embasar as abordagens das produções científicas em relação aos cuidados de enfermagem em pacientes com pneumonia associada à ventilação mecânica e, desta forma, foram dispostos no Quadro 2, de Contribuições dos estudos. 
Quadro 2 - Contribuições dos estudos.

\begin{tabular}{|c|c|c|c|c|}
\hline $\mathbf{N}^{\circ}$ & METODOLOGIA & OBJETIVO & RESULTADOS & $\begin{array}{c}\text { NÍVEL } \\
\text { DE } \\
\text { EVIDÊENCIA }\end{array}$ \\
\hline 01 & $\begin{array}{l}\text { Esse estudo testa o emprego } \\
\text { de uma estratégia educativa, } \\
\text { baseados em evidências, } \\
\text { entrevistando } 7 \text { enfermeiros e } \\
28 \text { técnicos assistencialistas de } \\
\text { uma unidade de terapia } \\
\text { intensiva. }\end{array}$ & $\begin{array}{l}\text { Determinar a eficácia de } \\
\text { estratégia educativa para } \\
\text { melhorar o desempenho da } \\
\text { equipe de enfermagem na } \\
\text { realização de } \\
\text { procedimentos preventivos } \\
\text { da pneumonia associada à } \\
\text { ventilação mecânica. }\end{array}$ & $\begin{array}{l}\text { Os resultados mostram que a } \\
\text { intervenção educativa teve } \\
\text { eficácia para a realização } \\
\text { correta da montagem do } \\
\text { VM com técnica asséptica, a } \\
\text { higienização da língua e a } \\
\text { manutenção da ordem correta } \\
\text { tubo-nariz-boca durante o } \\
\text { procedimento de higiene } \\
\text { brônquica. }\end{array}$ & 4 \\
\hline 02 & $\begin{array}{l}\text { Pesquisa qualitativa e } \\
\text { descritiva realizada em uma } \\
\text { unidade de terapia intensiva. }\end{array}$ & $\begin{array}{l}\text { Avaliar a adesão dos } \\
\text { profissionais de saúde a um } \\
\text { conjunto de boas práticas } \\
\text { de prevenção de } \\
\text { Pneumonia Associada à } \\
\text { Ventilação } \\
\text { Mecânica, índice de } \\
\text { conformidade às medidas } \\
\text { individuais e associação de } \\
\text { características clínicas dos } \\
\text { pacientes e adesão ao } \\
\text { conjunto de boas práticas } \\
\text { com a pneumonia. }\end{array}$ & $\begin{array}{l}\text { A avaliação da adesão às } \\
\text { boas práticas embasadas em } \\
\text { evidências científicas, o } \\
\text { estabelecimento } \\
\text { indicadores de resultado, } \\
\text { avaliação estrutural, educação } \\
\text { e vigilância de processos são } \\
\text { fundamentais para a } \\
\text { prevenção de IRAS. }\end{array}$ & 4 \\
\hline 03 & $\begin{array}{l}\text { Estudo } \\
\text { longitudinal de análise de } \\
\text { adesão ao bundle após } \\
\text { reestruturação, realizado em } \\
\text { UTI geral de adultos, entre } \\
\text { maio e novembro de } 2017 . \\
\text { Amostra por conveniência } \\
\text { com 154 pacientes em } \\
\text { ventilação mecânica. }\end{array}$ & $\begin{array}{l}\text { Avaliar a taxa de adesão } \\
\text { das ações preventivas da } \\
\text { equipe de enfermagem para } \\
\text { PAV, após a reestruturação } \\
\text { e aplicação do protocolo de } \\
\text { prevenção e verificar as } \\
\text { taxas de densidade de } \\
\text { incidência de pacientes } \\
\text { com PAV }\end{array}$ & $\begin{array}{l}\text { A média da taxa de adesão } \\
\text { das medidas preventivas em } \\
1.296 \text { avaliações realizadas } \\
\text { evidenciou adequação em: } \\
94 \% \text { posição do filtro; } 88,7 \% \\
\text { cabeceira elevada; } 77,3 \% \\
\text { higiene oral com clorexidina } \\
0,12 \% \text {; e } 91,7 \% \text { controle da } \\
\text { pressão do } \text { cuff. }\end{array}$ & 4 \\
\hline 04 & $\begin{array}{l}\text { Estudo transversal e } \\
\text { quantitativo, com amostra de } \\
90 \text { pacientes internados em um } \\
\text { hospital referência em } \\
\text { atendimento de doenças } \\
\text { cardiopulmonares, situado na } \\
\text { cidade de Fortaleza/Ceará. } \\
\text { Coleta de dados realizada em } \\
\text { outubro de } 2016 \text { através de um } \\
\text { instrumento estruturado. }\end{array}$ & $\begin{array}{l}\text { Descrever as características } \\
\text { clínicas de pacientes sob } \\
\text { ventilação r mecânica } \\
\text { internados em Unidade de } \\
\text { Terapia Intensiva(UTI). }\end{array}$ & $\begin{array}{lr}\text { Os principais motivos de } \\
\text { internação foram as doenças } \\
\text { cardiovasculares } & (53,3 \%) \\
88,9 \% & \text { utilizaram } \\
\text { antibioticoterapia; } & 34,4 \% \\
\text { apresentaram } & \text { pneumonia } \\
\text { associada à } & \text { ventilação } \\
\text { mecânica; 80\% } & \text { utilizaram } \\
\text { drogas vasoativas e apenas } & \text { e apen } \\
25,6 \% & \text { realizaram } \\
\text { traqueostomia } & \text { após a } \\
\text { internação 36,7\%. }\end{array}$ & 4 \\
\hline 05 & $\begin{array}{l}\text { Pesquisa de delineamento } \\
\text { transversal, de cunho quanti- } \\
\text { qualitativo. Participaram da } \\
\text { pesquisa } 28 \text { profissionais de } \\
\text { saúde. }\end{array}$ & \begin{tabular}{lrr} 
Avaliar o conhecimento \\
dos profissionais de saúde \\
sobre a questão que \\
envolve a prevenção da \\
(PAVM) em pacientes \\
críticos internados nas \\
(UTIs), com vista a \\
promover & \multicolumn{2}{c}{ educação } \\
permanente & (EP) para \\
profissionais das UTIs.
\end{tabular} & $\begin{array}{l}43 \% \text { confirmaram possuir } \\
\text { conhecimento sobre bundle } \\
\text { de prevenção; } 36 \% \text { citaram já } \\
\text { terem participado de algum } \\
\text { treinamento sobre a temática; } \\
96 \% \text { manifestou interesse em } \\
\text { receber algum treinamento } \\
\text { específico; apenas } 25 \% \\
\text { responderam corretamente a } \\
\text { pressão ideal do cuff; } 96 \% \\
\text { afirmou avaliar, diariamente, } \\
\text { a retirada da sedação. }\end{array}$ & 4 \\
\hline & $\begin{array}{llr}\text { Estudo transversal } & \text { e } \\
\text { quantitativo, com amostra de } \\
90 \text { pacientes internados em um } \\
\text { hospital referência } \quad \text { em }\end{array}$ & $\begin{array}{l}\text { Descrever as características } \\
\text { clínicas de pacientes sob } \\
\text { ventilação } r \text { mecânica } \\
\text { internados em Unidade de }\end{array}$ & $\begin{array}{l}\text { Os principais motivos de } \\
\text { internação foram as doenças } \\
\text { cardiovasculares } \quad(53,3 \%) \text {; } \\
88,9 \% \text { utilizaram }\end{array}$ & \\
\hline
\end{tabular}




\begin{tabular}{|c|c|c|c|c|}
\hline 06 & $\begin{array}{l}\text { atendimento de doenças } \\
\text { cardiopulmonares, situado na } \\
\text { cidade de Fortaleza/Ceará. } \\
\text { Coleta de dados realizada em } \\
\text { outubro de } 2016 \text { através de um } \\
\text { instrumento estruturado. }\end{array}$ & Terapia Intensiva (UTI). & 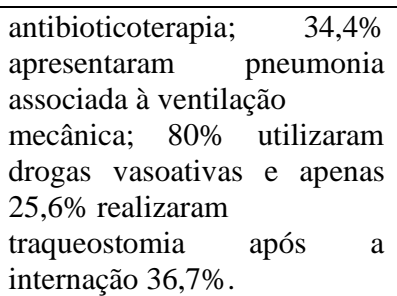 & 4 \\
\hline 07 & $\begin{array}{l}\text { Estudo observacional com } 13 \\
\text { pacientes do sexo masculino. } \\
\text { Foi aplicada a escala de Acute } \\
\text { Physiology and Chronic } \\
\text { Health Evalution e o Clinical } \\
\text { Pulmonary Infection Score } \\
\text { após } 72 \text { horas de internação } \\
\text { orotraqueal. }\end{array}$ & $\begin{array}{l}\text { Verificar a prevalência da } \\
\text { pneumonia associada à } \\
\text { ventilação mecânica por } \\
\text { meio da coleta e análise das } \\
\text { secreções } \\
\text { traqueobrônquicas. }\end{array}$ & $\begin{array}{l}\text { Observou-se uma pontuação } \\
\text { média de } 33 \text { pontos, ou seja, } \\
\text { estes pacientes apresentaram } \\
\text { em média } 75 \% \text { de chance de } \\
\text { óbito. Foram encontradas } \\
\text { como microorganismos de } \\
\text { maior prevalência o o } \\
\text { Staphylococcus aureus } \\
(23,07 \%) \quad e \quad \text { Klebsiella } \\
\text { pneumoniae }(15,38 \%) \text {. }\end{array}$ & 4 \\
\hline 08 & 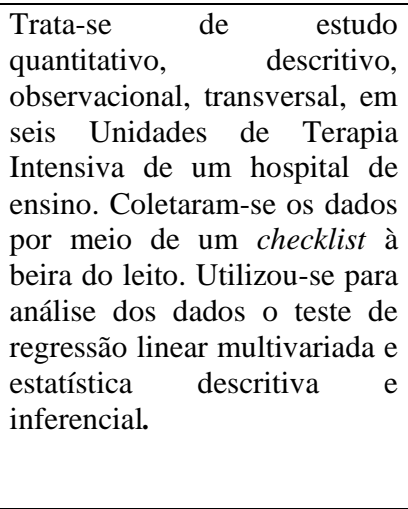 & $\begin{array}{l}\text { Conhecer a adesão da } \\
\text { equipe de enfermagem ao } \\
\text { protocolo de pneumonia } \\
\text { associada à ventilação } \\
\text { mecânica nas Unidades de } \\
\text { Terapia Intensiva. }\end{array}$ & $\begin{array}{l}\text { A equipe de enfermagem } \\
\text { manteve em acordo com o } \\
\text { protocolo institucional } \\
\text { aproximadamente } 90,05 \% \\
\text { das cabeceiras elevadas de } \\
30^{\circ} \text { a } 45^{\circ} \text {. Demonstrou-se } \\
\text { estaticamente a relação PAV } \\
\text { com o sexo masculino, tempo } \\
\text { de internação nos primeiros } \\
15 \text { dias e filtro HME. Houve } \\
\text { a correlação de cabeceira } \\
\text { elevada com idade e tempo } \\
\text { de internação e filtro HME } \\
\text { com tempo de internação. }\end{array}$ & 4 \\
\hline 09 & $\begin{array}{l}\text { Trata-se de } \\
\text { bibliográfico, tipo revisão } \\
\text { integrativa, em que os artigos } \\
\text { consultados foram nas Bases } \\
\text { de Dados Lilacs, Medline a } \\
\text { Biblioteca Virtual Scielo. } \\
\text { Informam-se que a busca foi } \\
\text { realizada em janeiro de } 2018 \text { e } \\
\text { amostra final de } 20 \text { artigos, } \\
\text { apresentando-se os resultados } \\
\text { em figura. }\end{array}$ & $\begin{array}{lrr}\text { Analisar } & \text { os } & \text { principais } \\
\text { fatores de } & \text { risco que } \\
\text { favorecem à } & \text { pneumonia } \\
\text { associada } & \text { à } & \text { ventilação } \\
\text { mecânica. } & & \end{array}$ & $\begin{array}{l}\text { Observou-se a importância da } \\
\text { atenção do enfermeiro com } \\
\text { pacientes que se encontrem } \\
\text { em ambiente de terapia } \\
\text { intensiva e necessitem da } \\
\text { ventilação mecânica a fim de } \\
\text { prevenir, diagnosticar e tratar } \\
\text { uma pneumonia associada à } \\
\text { ventilação mecânica. }\end{array}$ & 4 \\
\hline 10 & $\begin{array}{l}\text { É uma pesquisa de abordagem } \\
\text { descritiva e exploratória, } \\
\text { qualitativa. } \\
\text { Os sujeitos foram sete } \\
\text { enfermeiros da UTI de um } \\
\text { hospital universitário na } \\
\text { cidade de Salvador, Bahia, } \\
\text { durante o mês de outubro de } \\
2010 \text { Os dados foram } \\
\text { coletados através de entrevista } \\
\text { semiestruturada. }\end{array}$ & $\begin{array}{l}\text { Este estudo descreve as } \\
\text { medidas conhecidas pelas } \\
\text { enfermeiras de cuidados } \\
\text { intensivos para prevenir } \\
\text { PAV. }\end{array}$ & $\begin{array}{l}\text { A aspiração endotraqueal é a } \\
\text { única medida mencionada por } \\
\text { todos os entrevistados; trocar } \\
\text { o circuito de ventilação, } \\
\text { higiene oral com clorexidina, } \\
\text { reduziram o tempo de } \\
\text { ventilação mecânica; } \\
\text { elevação da cabeceira da } \\
\text { cama e lavar as mãos foram } \\
\text { citados com menor } \\
\text { frequência pelos participantes } \\
\text { da pesquisa. }\end{array}$ & 4 \\
\hline \multirow[t]{2}{*}{11} & $\begin{array}{l}\text { Trata-se de um estudo } \\
\text { qualitativo, descritivo e } \\
\text { exploratório realizado em um } \\
\text { hospital privado de pequeno } \\
\text { porte. Compôs-se de uma } \\
\text { amostra intencional com sete } \\
\text { profissionais de Enfermagem e } \\
\text { efetuou-se a coleta de dados } \\
\text { por meio de entrevistas } \\
\text { semiestruturadas e gravadas. } \\
\text { Utilizou-se a análise temática } \\
\text { dos dados. }\end{array}$ & $\begin{array}{l}\text { Apreender a percepção dos } \\
\text { profissionais de } \\
\text { Enfermagem sobre a } \\
\text { segurança do paciente sob } \\
\text { ventilação mecânica com } \\
\text { vistas à prevenção da PAV. }\end{array}$ & $\begin{array}{l}\text { Elaborou-se um mapa } \\
\text { temático composto pelo tema } \\
\text { "Risco de pneumonia } \\
\text { associada à ventilação } \\
\text { mecânica: percepção dos } \\
\text { profissionais } \\
\text { Enfermagem", que congrega } \\
\text { o subtema "Prevenção da } \\
\text { pneumonia associada à } \\
\text { ventilação mecânica: O que } \\
\text { fazem os profissionais de } \\
\text { Enfermagem?" }\end{array}$ & 4 \\
\hline & Estudo descritivo realizado em & Mensurar & $\mathrm{O}$ escore médio atribuído & \\
\hline
\end{tabular}




\begin{tabular}{|c|c|c|c|c|}
\hline 12 & $\begin{array}{l}\text { unidades de terapia intensiva } \\
\text { de um hospital universitário } \\
\text { por meio de questionário e } \\
\text { análise de registros. A amostra } \\
\text { foi constituída de } 47 \text { ( } 72,3 \%) \\
\text { entre } 65 \text { enfermeiros e foram } \\
\text { analisados } 53 \text { prontuários. }\end{array}$ & $\begin{array}{l}\text { enfermeiros a valoração da } \\
\text { higiene bucal de pacientes } \\
\text { adultos intubados, verificar } \\
\text { a identificação e registros } \\
\text { dos diagnósticos e } \\
\text { prescrições de enfermagem } \\
\text { pertinentes às alterações da } \\
\text { cavidade bucal e avaliar os } \\
\text { registros e as ações dos } \\
\text { técnicos de enfermagem } \\
\text { relacionados à higienização } \\
\text { bucal. }\end{array}$ & $\begin{array}{l}\text { pelos } \\
\text { relacionado à valoração do } \\
\text { procedimento foi de 83. A } \\
\text { maioria relata avaliar as } \\
\text { condições da cavidade bucal } \\
\text { e prescreve o procedimento } \\
\text { de higienização. Nos } \\
\text { prontuários não foram } \\
\text { encontrados os diagnósticos } \\
\text { de enfermagem relacionados. } \\
\text { Em 67\% dos prontuários } \\
\text { havia registros sobre a } \\
\text { realização da higiene pelos } \\
\text { técnicos. }\end{array}$ & 4 \\
\hline
\end{tabular}

Fonte: Autores.

Na pesquisa foram encontrados artigos relacionados à boas práticas do enfermeiro mediante a PAV. No total foram 5 artigos que abordavam tal temática com enfoque na utilização de protocolos bundles. Na utilização os artigos demonstram sua eficácia no agrupamento das melhores práticas assistenciais que, quando usados individualmente, podem não ter o desfecho desejado. Tal sucesso depende do envolvimento da equipe multiprofissional, principalmente a enfermagem, onde suas ações devem ser centradas na seleção das intervenções.

Outros três artigos possuem enfoque com base no cuidado de enfermagem, selecionado pelos enfermeiros como boas práticas, a literatura ressalta a necessidade do enfermeiro avaliar o paciente antes da aspiração, mas também durante e após a técnica, mediante exame físico e monitorização, a fim de assegurar um procedimento seguro e consequentemente a diminuição de erros.

Mediante levantamento dos 12 artigos da revisão integrativa, emergiram duas categorias que possibilitaram uma discussão em comum: Fatores de risco e medidas de prevenção à Pneumonia Associado à Ventilação Mecânica e Boas práticas do enfermeiro mediante a PAV.

\section{Discussão}

\subsection{Fatores de risco e medidas de prevenção à Pneumonia Associado à Ventilação Mecânica}

A grande maioria dos pacientes na UTI necessita de (VM). Consequentemente aumentam as chances do paciente contrair a (PAV). As infecções destacadas em (UTI) estão relacionadas a cadeia de erros durate a prevenção de agravos, ao sistema de monitorização, erros mediante ao processo de indicar, colocar, manter e retirar os dispositivos tubulares (Melo et al., 2019).

A enfermagem deve estar preparada para prevenir os riscos da PAV. Uma das alternativas é a adoção de protocolos bundles haja vista que seu objetivo é reduzir a falta de informações, melhorar a conduta profissionais da enfermagem e promover a prevenção (Zigart et al., 2019). Todavia, os protocolos isoladamente não asseguram prevenção e redução da PAV pois é necessário um conjunto de ações juntamente com bundles para alcançar o objetivo (Barros, 2019). O enfermeiro está diretamente inserido na assistência aos pacientes, cuidados e prevenção (Dutra et al., 2019).

Os bundles são uma série de medidas preventivas que visam evitar infecções em ambientes hospitalares criado pelo Institute for Healthcare Improvement (IHI). Os bundles tornaram-se um conjunto de boas práticas que possibilitam um melhor cuidado com o paciente proporcionando melhores resultados de prevenção de doenças e melhorando o bem-estar de pacientes. Tais conjuntos de medidas são baseados em pesquisas científicas que focam nos principais fatores de risco associados ao desenvolvimento da PAV em pacientes em ventilação artificial (Shimabukuro et al., 2014).

Existem variados fatores de risco que aumentam os números de casos de PAV em UTI's. Tais fatores são classificados como: não modificáveis e modificáveis. Os não modificáveis são aqueles não controlados pelos profissionais da saúde, entre eles estão: idade; coma; choque; gravidade da doença; antecedência de Doença Pulmonar Obstrutiva Crônica; etc. 
Já os fatores de risco modificáveis já aquele ao qual a equipe de enfermagem pode intervir afim de alterar a situação de risco e assim prevenir a PAV, entre esses fatores estão: tempo prolongado de ventilação mecânica maior que sete dias; aspirado do condensado contaminado dos circuitos do ventilador; contaminação exógena; colonização microbiana da própria UTI; cirurgias prolongadas; aspiração de secreções contaminadas, etc. (Santos et al. 2018). Na modificação desses fatores de riscos destaca-se algumas medidas.

A prevenção diminui as chances de infecção e com isso, reduz a utilização de antibióticos que pode ocasionar uma seletividade de bactérias da PAV resistente aos medicamentos. Além disso, diminui o tempo de permanência do paciente na UTI e evita a exposição a outros tipos de infecção (Moreira et al., 2011).

O artigo de Ribeiro (2018) aponta os aspectos clínicos dos pacientes sob ventilação mecânica. Tal conhecimento proporciona ao enfermeiro os conhecimentos técnico-científicos necessários para melhor cuidado visando minimizar os efeitos adversos causados pela ventilação mecânica. Já o artigo de Ferreira (2017) comenta sobre a prevalência de PAV em pacientes do sexo masculino utilizando a técnica de análise de secreções traqueobrônquicas. A identificação das bactérias Klebsiella pneumoniae e Staphylococcus aureus tornou-se útil para um melhor tratamento.

Nos estudos de Santos et al. (2018) e Zanei et al. (2016), estão contidos os fatores de risco de maior prevalência na PAV. Especificamente em Santos et al. (2018) é apresentado os fatores em um aspecto geral promovendo uma discussão acerca do surgimento dessa patologia, além de aponta soluções para os fatores de risco modificáveis.

O paciente ao permanecer por um tempo prolongado com a ventilação mecânica desenvolve uma alteração no mecanismo de defesa dos pulmões, ocasionando uma oportunidade para ocorrer a PAV (Moreira et al., 2011). Uma medida de modificação está na redução no número de dias de intubação por ventilação mecânica dado que reduz a aspiração de secreçães contaminadas devido à redução do reflexo de tosse (Dutra et al., 2019).

Existem medidas consideradas simples de prevenção da PAV, porém ainda negligenciadas por profissionais da saúde, entre eles está a lavagem das mãos considerada importante na prevenção uma vez que evitam a contaminação exógenas de pacientes que estão em situação de vulnerabilidade na UTI. A alimentação em pacientes em posição supina a $0^{\circ}$ é um fator de risco para pacientes ventilados mecanicamente, e a elevação da cabeceira em $30^{\circ}$ a $45^{\circ}$ é uma das alternativas que devem ser usadas pela equipe de enfermagem (Moreira et al., 2011).

Em Zanei et al. (2016) o enfoque principal é a higiene oral dos pacientes sob ventilação mecânica, e nesse estudo, é reconhecido pelos enfermeiros a importância de tal profilaxia na prevenção da PAV. Porém, em ambos os estudos é verificado a falta de capacitação dos enfermeiros quanto a associar medidas preventivas aos fatores de risco.

A realização da higiene oral do paciente diminui a incidência de PAV. Segundo Zanei et al, a utilização de antisséptico vem sendo defendido como umas das grandes medidas para o baixo desenvolvimento da PAV. Porém, para o mesmo autor, ainda é pouco utilizada pela equipe de enfermagem. Segundo estudos americanos, essa negligencia com a higiene bucal está relacionada na prioridade dos enfermeiros para outras tipos de prevenção.

A PAV contém seus fatores de riscos que podem ser modificados pela equipe de enfermagem e a adoção de protocolos bundles fundamentados em pesquisas cientificas visa a prevenção possibilitando uma melhor saúde dos pacientes em UTI's.

\subsection{Boas práticas do enfermeiro mediante a PAV}

Pessoas que carecem do uso de Ventilação Mecânica (VM) são frequentes em UTI's. A Pneumonia associada à Ventilação Mecânica (PAV) é a segunda infecção nosocomial mais frequente entre os pacientes ventilados. Além de doença, a PAV é considerada um marcador da qualidade de assistência à saúde por ser uma doença sujeita a prevenção (Nascimento, Farias \& Souza, 2019). 
As Infecções Relacionadas à Assistência à Saúde (IRAS) são consideradas como um problema de saúde pública por serem conceituadas como eventos adversos adquiridos durante a prestação dos cuidados e, por isso, se apresentam como riscos significativos à segurança do paciente (Ferreira et al., 2017).

A ventilação mecânica substitui total ou parcial a ventilação espontânea, na qual é muito indicada na insuficiência respiratória aguda (IRA) ou crônica. Quando empregada de modo invasivo é utilizado tubo endotraqueal ou cânula de traqueostomia. Esse tipo de suporte demanda assistência especializada de uma equipe multiprofissional, tendo o enfermeiro a maior responsabilidade em manter a permeabilidade das vias áreas do paciente intubado, possuir o domínio sobre os parâmetros do ventilador e avaliar a adaptação do paciente aos parâmetros e assim implantar os cuidados de enfermagem (Santos et al., 2018).

Os cuidados considerados como boas práticas assistenciais nesse contexto devem ser adquiridas, com o intuito de promover um serviço qualificado visando a segurança deste paciente. Na enfermagem, entende-se como boas práticas um conjunto de relações mútuas e indissociável de teorias, técnicas e atividades visto como as melhores opções disponíveis para o cuidado de cada paciente, através de conhecimentos, objetivos e evidências no interesse da saúde (Santos et al., 2018).

Com isso, destaca-se a construção de pacote de intervenções, denominado bundles, que se apresenta como um pacote de medidas baseadas em evidências científicas integradas para prática de prevenções dentro da assistência em saúde (Ribeiro et al., 2018).

Os protocolos são instrumentos de medidas desenvolvidos, que facilitam a disseminação de práticas eficazes e a redução de erros, podendo diferir entre si quanto ao formato e método de desenvolvimento, porém, devem estar sempre baseados em evidências científicas (Santos et al., 2018).

Desta forma destaca-se a gênese de Tecnologias Educacionais (TE) em saúde com um olhar voltado para a equipe inter e multiprofissional, certo de que, por meio destas tecnologias, é possível visualizar melhor informações, potencializando o conhecimento, atuando no processo de ensino-aprendizagem na educação permanente do profissional em saúde (Alecrim et al., 2019).

A enfermagem como um todo é a maior responsável pela assistência aos pacientes em UTI, são os que mais conhecem o que necessário a cada paciente e estão em contato direto e contínuo, fazendo-se imprescindível no cuidado prestado, o conhecimento teórico-prático são fundamentais para prevenir e minimizar a incidência desses acometimentos, como a PAV (Nascimento, Farias \& Souza, 2019).

\section{Conclusão}

Conclui-se que a equipe que compõe a enfermagem e que atua frente à PAV exerce um papel de extrema importância para o cuidar assistencial de enfermagem nas UTI's. Pois os "Fatores de riscos modificáveis na prevenção à PAV" necessitam de profissionais que proporcione ao paciente através do seu conhecimento teórico-prático, os cuidados específicos que precisam ser realizados rotineiramente, visando a saúde do paciente.

A implementação de práticas adequadas, associado à educação da equipe multiprofissional é um fator determinante para a redução das taxas de incidências, na redução do tempo de internação nas UTI’s, além de promover uma assistência segura. É necessário que seja dado grande ênfase a questão da melhoria no processo assistencial, que práticas educativas sejam constantes entre as equipes, que os cuidados possam efetivamente se aumentados, diminuindo proporcionalmente a incidência da PAV, melhorando a qualidade assistencial e a segurança do paciente.

Dentro desta questão o enfermeiro possui uma maior responsabilidade pela assistência prestada aos pacientes de UTI, são eles quem mais conhecem as peculiaridades de cada ator. Dessa forma, seu conhecimento torna-se imprescindível, pois são eles que com sua assistência previnem e diminuem a taxa da PAV. 
Espera-se que este estudo contribua para o controle de infecções relacionadas à assistência em saúde nas UTI's e que sirva para subsidiar trabalhos futuros relacionados à este assunto. Compreendendo o embasamento técnico-prático sobre a prevenção e cuidados relacionados à pneumonia associada à ventilação mecânica.

\section{Referências}

Alecrim, R. X. et al. (2019). Estratégias para prevenção de pneumonia associada à ventilação mecânica: revisão integrativa. Revista Brasileira de Enfermagem. 72(2), 521-30.

Bardin, L. Análise de conteúdo. Lisboa: Edições 70, 2000.

Barros, F. R. B. (2019). Adesão ao bundle de prevenção de pneumonia associada à ventilação mecânica. Revista Cuidarte, 10 (2), e746.

Dutra, L. A. et al. (2019). Pneumonia associada à ventilação mecânica: percepção dos profissionais de enfermagem. Revista de enfermagem UFPE On Line. Recife. 13, 884-892.

Farias, G. M. D. et al. (2009). Pacientes sob ventilação mecânica: cuidados prestados durante a aspiração endotraqueal. Revista Cientifica Internacional. 2(9).

Ferreira, E. G. et al. (2017). Prevalência de pneumonia associada à ventilação mecânica por meio de análise das secreções traqueobrônquicas. Revista Rene. $18,114-120$.

Galvão, T. F., Pansani, T. de S. A. \& Harrad, D. (2015). Principais itens para relatar Revisões sistemáticas e Meta-análises: A recomendação PRISMA. Epidemiologia e Serviços de Saúde [online]. 24(2), 335-342.

Gonçalves, F. A. F. et al. (2012). Eficácia de estratégias educativas para ações preventivas da pneumonia associada à ventilação mecânica. Revista de Enfermagem Anna Nery. 16(4), 802-808.

Lourençone, E. M. S. et al. (2019). Adesão às medidas preventivas versus incidência de pneumonia associada à ventilação mecânica. Revista Epidemiologica Controle Infecção Santa Cruz do Sul. 9, 142-148.

Melo, M. M. et al. (2019). Pneumonia Associada à Ventilação Mecânica: Conhecimento dos Profissionais de Saúde Acerca da Prevenção e Medidas Educativas. Rev Fund Care Online. 11(2, n. esp), 377-382.

Mendes, K. D. S., Silveira, R. C. de C. P. \& Galvão, C. M. (2019). Use of the bibliographic reference manager in the selection of primary studies in integrative reviews. Texto e Contexto Enfermagem, 28, 1-13.

Minayo, M. C. S. (2001). Ciência, Técnica E Arte: O Desafio Da Pesquisa Social. Minayo, M.C.S (Org.). Pesquisa Social: teoria,método e criatividade, 80.

Moreira, B. S. G. et al. (2011). Pneumonia associada à ventilação mecânica: medidas preventivas conhecidas pelo enfermeiro. Revista baiana de enfermagem. $25,99-106$

Neto, J. D. A. et al. (2016). Profissionais de saúde da unidade de terapia intensiva: percepção dos fatores restritivos da atuação multiprofissional. Revista Brasileira em promoção da Saúde, 29(1), 43-50.

Prazeres, L. E. N. dos. et al. (2021). Atuação do enfermeiro nos cuidados em Unidades de Terapia Intensiva Neonatal: Revisão integrativa da literatura. Research, Society and Development, 10(6), e1910614588. DOI: https://doi.org/10.33448/rsd-v10i6.14588.

Ribeiro, C. M. et al., (2018). Caracterização clínica dos pacientes sob ventilação mecânica internados em unidade de terapia intensiva. J. res.: fundam. care. online. 10(2), 496-502.

Rodrigues, A. N. et al. (2016). Impactos e fatores determinantes no bundle de pneumonia associada à ventilação mecânica. Revista Brasileira de Enfermagem, 69(6), 1108-1114.

Santos, C, R. et al. (2018). Fatores de risco que favorecem a pneumonia associada à ventilação mecânica. Revista de enfermagem UFPE On Line. 12(12), 3401-3415.

Shimabakuro, P. M. S. et al. (2014). Implantação de Bundles em Unidade de Terapia Intensiva: Um relato de experiência. Revista de Enfermagem da UFSM, $4(1), 227-236$.

Silva, T. G. D. et al. (2017). Incidence of ventilator-associated pneumonia in an intensive care unit Incidência de pneumonia associada à ventilação mecânica em uma Unidade de Terapia Intensiva. Revista de Pesquisa: Cuidado é Fundamental Online, 9(4), 1121.

Soares, C. B. et al. (2014). Revisão Integrativa versus Revisão Sistemática. Reme: Revista Mineira de Enfermagem, 12(4), 758-764.

Souza, M. T. de, Silva, M. D. da \& Carvalho, R. de. (2010). Integrative review: what is it? How to do it? Einstein (São Paulo) [online]. 8(1), $102-106$.

Teixeira, E. et al. (2013). Integrative literature review step-by-step \& convergences with other methods of review. Rev Enferm UFPI, Teresina, 2, 3-7.

Tubert-Brohman, I. et al. (2013). Improved Docking of Polypeptides with Glide. Journal of Chemical Information and Modeling. 53(7), 1689-1699.

Ursi, E. S. \& Galvão, C. M. (2006). Prevenção de lesões de pele no perioperatório: revisão integrativa da literatura. Rev Latino-am Enfermagem, 14(1), 12431 . 
Research, Society and Development, v. 10, n. 7, e58210716935, 2021

(CC BY 4.0) | ISSN 2525-3409 | DOI: http://dx.doi.org/10.33448/rsd-v10i7.16935

Zanei, S. S. V. et al. (2016). Valorização e registro sobre a higiene oral dos pacientes intubados nas unidades de terapia intensiva. REME - Rev Min Enferm. 20,1-8.

Zigart, J. A. A. et al. (2019). Adesão ao protocolo de pneumonia associado à ventilação mecânica. Revista de enfermagem UFPE On Line, $13,655-663$. 